Afrika Statistika

Afrika Statistika

Vol. 11(2), 2016, pages 1061-1074.

DOI: http://dx.doi.org/10.16929/as/2016.1061.92

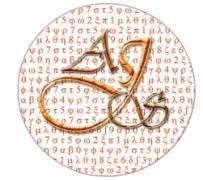

ISSN 2316-090X

\title{
Robust bayesian inference of generalized Pareto distribution
}

\author{
Fatiha Mokrani ${ }^{1}$, Hocine Fellag ${ }^{1, *}$ and Abdelhakim Necir ${ }^{2}$ \\ ${ }^{1}$ Laboratory of Pure and Applied Mathematics, Mouloud Mammeri University, Tizi-Ouzou, Algeria \\ ${ }^{2}$ Laboratory of Applied Mathematics, Mohamed Khider University, Biskra, Algeria
}

Received August 1, 2016; Accepted December 20, 2016

Copyright(c) 2016, Afrika Statistika and Statistics and Probability African Society (SPAS). All rights reserved

\begin{abstract}
In this work, robust Bayesian estimation of the generalized Pareto distribution is proposed. The methodology is presented in terms of oscillation of posterior risks of the Bayesian estimators. By using a Monte Carlo simulation study, we show that, under a suitable generalized loss function, we can obtain a robust Bayesian estimator of the model.

Résumé. Dans ce travail, nous présentons une analyse de robustesse Bayesienne des estimateurs des paramètres d'un modèle de Pareto généralisé en termes d'oscillation des risques a posteriori. En utilisant une étude exhaustive de Monte Carlo, nous prouvons que, moyennant une fonction perte généralisée adéquate, on peut construire un estimateur Bayesien robuste du modèle.
\end{abstract}

Key words: Bayesian estimation; Extreme value; Generalized Fisher information; Generalized Pareto distribution; Monte Carlo; Robustness.

AMS 2010 Mathematics Subject Classification : 62F15; 62F35

\section{Introduction}

In the extreme value theory, the Generalized Pareto Distribution (GPD) play an important role, especially in practical situations. In fact, applications of the GPD to areas such as insurance, reliability, finance, meteorology and environment are widely spread out in the literature. The GPD was first explicitly introduced by Pickands (1975) in the extreme value framework.

Arnold and Press (1989) may be considered as the pioneers in using Bayesian techniques for parameter estimation in the extreme value context. They recommend (when appropriate)

*Corresponding author : hefellag@yahoo.com

Fatiha Mokrani : fatiha_mokrani@yahoo.fr

Abdelhakim Necir: necirabdelhakim@yahoo.fr 
the use of conjugate prior distributions for the parameters at hand. However, one can notice that very few number of authors treated the topic of the Bayesian estimation of GPD. That's why, until now, one can say that the number of applications developed in the extreme value context is still relatively modest.

The great majority of the existing approaches dealing with Bayesian procedures are focused on the estimation of the parameters of Pareto distribution. De Zea Bermudez and Turkmann (2003) suggested a method for estimating the parameters of GPD, that uses the Pareto and the gamma distributions as priors for the parameters. Diebolt et al. (2005) addressed this issue by making use of the quasi-conjugate distributions approach. A nice review on robust estimation of GPD parameters by means of the Bayesian methodology may be found in De Zea Bermudez and Kotz (2010).

Robust Bayesian Analysis (Berger et al., 2000) is concerned with the sensitivity of Bayesian analysis results to the inputs for the analysis. In Robust Bayesian analysis a prior is assumed to belong to a family of distributions, instead of being specified definitely and this leads to a collection of Bayes actions. There are many approaches in Bayesian robustness context, see, e.g., Micheas (2006).

In this work, we propose to use a suitable generalized loss function to perform robustness and sensitivity analysis of the Bayesian estimators according to the methodology proposed by Męczarski and Zieliński (1991). The paper is outlined as follows. The second section presents the genesis of the model. The Bayesian robustness analysis of this model is preformed in the third section. In this part of the paper, Bayesian stability of the two parameters of the model is studied under a generalized quadratic loss function. The last section is devoted to an exhaustive simulation study where the performance of the Bayesian estimators is analyzed.

\section{Genesis of the model}

Consider $\left(X_{1}, X_{2}, \ldots, X_{n}\right)$ a random sample from $\operatorname{GPD}(\alpha, \beta)$ with density

$$
f(x, \alpha, \beta)=\frac{\alpha}{\beta}\left(1+\frac{x}{\beta}\right)^{-\alpha-1} \quad x \geq 0, \quad \text { with } \quad \alpha>0 \quad \text { and } \quad \beta>0 .
$$

The likelihood function based on the observation $x=\left\{x_{1}, x_{2}, \ldots, x_{n}\right\}$ is

$$
L(x, \alpha, \beta)=\left(\frac{\alpha}{\beta}\right)^{n} \exp \left\{-(\alpha+1) \sum_{i=0}^{n} \ln \left(1+\frac{x_{i}}{\beta}\right)\right\}
$$

Assume that $\pi_{0}(\alpha, \beta)$ is the prior of the parameters $(\alpha, \beta)$. Then, using the Bayes rule, we get the corresponding posterior distribution

$$
\pi(\alpha, \beta \mid x)=\frac{L(x ; \alpha, \beta) \cdot \pi_{0}(\alpha, \beta)}{\int_{0}^{+\infty} \int_{0}^{+\infty} L(x ; \alpha, \beta) \cdot \pi_{0}(\alpha, \beta) d \alpha d \beta}
$$

Generally, there is no tractable expression of this posterior density and then, the computation of the Bayesian estimators can be done using Monte Carlo procedures only. In the following, we will perform robust Bayesian analysis of this estimation using the procedure proposed by Męczarski and Zieliński (1991). This method is detailed in the next section.

Journal home page: www.jafristat.net ; www.projecteuclid.org/as 


\section{Bayesian robustness}

\subsection{Basic quadratic loss function}

\subsubsection{Bayesian stability for $\alpha$}

Consider the following priors for $\alpha$ and $\beta$ respectively,

$$
\alpha \sim \operatorname{Gamma}\left(a_{0}, b_{0}\right), \quad a_{0}>0, \quad b_{0}>0 \quad \text { and } \quad \beta \sim \text { non informative. }
$$

Then, the priors densities of $\alpha$ and $\beta$ are as follows:

$$
\begin{aligned}
& \pi_{0}(\alpha)=\frac{b_{0}^{a_{0}} \alpha^{a_{0}-1} e^{-\alpha b_{0}}}{\Gamma\left(a_{0}\right)} \\
& \pi_{1}(\beta)=\frac{1}{\beta}, \quad \beta>0 .
\end{aligned}
$$

The joint posterior distribution of the parameters $\alpha$ and $\beta$ is given through Bayes theorem by:

$$
\pi(\alpha, \beta \mid x)=\frac{\pi_{0}(\alpha) \pi_{1}(\beta) L(x, \alpha, \beta)}{\int_{0}^{\infty} \int_{0}^{\infty} \pi_{0}(\alpha) \pi_{1}(\beta) L(x, \alpha, \beta) d \alpha d \beta}
$$

which is usually expressed in a simpler way as:

$$
\pi(\alpha, \beta \mid x) \propto \pi_{0}(\alpha) \pi_{1}(\beta) L(x, \alpha, \beta)
$$

Then

$$
\pi(\alpha, \beta \mid x) \propto \alpha^{n+a_{0}-1} \beta^{-n} \exp \left\{-\alpha b_{0}-(\alpha+1) \sum_{i=0}^{n} \ln \left(1+\frac{x_{i}}{\beta}\right)\right\}
$$

However, the marginal posterior distributions of the parameters $\alpha$ and $\beta$ do not correspond to known distributions. To this purpose, we use Markov chain Monte Carlo (MCMC) methods, especially, Metropolis-Hastings algorithm within a Gibbs sampling (Gilks et al , 1986) in order to obtain samples from the marginal distributions of the parameters.

Gibbs sampling with Metropolis-Hastings step

First, we propose to derive the conditional distributions for each parameter. Then, we obtain the following densities

$$
\pi(\alpha \mid \beta, x) \propto \alpha^{n+a_{0}-1} \exp \left\{-\alpha\left(b_{0}-\sum_{i=0}^{n} \ln \left(1+\frac{x_{i}}{\beta}\right)\right)\right\}
$$

and

$$
\pi(\beta \mid \alpha, x) \propto \beta^{-n} \exp \left\{-(\alpha+1) \sum_{i=0}^{n} \ln \left(1+\frac{x_{i}}{\beta}\right)\right\}
$$

One can notice that the conditional distribution

$$
\alpha \mid \beta \sim \operatorname{Gamma}\left(n+a_{0}, b_{0}-\sum_{i=0}^{n} \ln \left(1+\frac{x_{i}}{\beta}\right)\right)
$$

However, $\beta \mid \alpha$ is not known. That's why, in this case, we will use the method of MetropolisHastings. Finally, the algorithm is as follows

Journal home page: www.jafristat.net ; www.projecteuclid.org/as 
- get the initial value $\left(\alpha^{(1)}, \beta^{(1)}\right)$

(1) set an initial value $\beta^{(1)}$

(2) generate $\alpha^{(1)} \mid \beta^{(1)}$ from Gamma $\left(n+a_{0}, b_{0}+\sum_{i=1}^{n} \ln \left(1+\frac{x_{i}}{\beta^{(1)}}\right)\right)$

- For $k=2, \ldots, N$ get the couples $\left(\alpha^{(2)}, \beta^{(2)}\right), \ldots,\left(\alpha^{(N)}, \beta^{(N)}\right)$

(1) generate $\beta^{(k)} \mid \alpha^{(k-1)}$ with Metropolis-Hastings algorithm

- pick a proposal density $q(y)=y e^{-\frac{2}{\beta} y}$ and generate $y \sim \operatorname{Gamma}\left(2 ; \frac{2}{\beta}\right)$

- compute $\rho=\min (1, r)$ where

$$
r=\frac{\pi\left(y \mid \alpha^{(k-1)}\right) q\left(\beta^{(k-1)}\right)}{\pi\left(\beta^{(k-1)} \mid \alpha^{(k-1)}\right) q(y)}
$$

take $\beta^{(k)}=y$ with probabiliy $\rho$ and $\beta^{(k)}=\beta^{(k-1)}$ else

(2) generate $\alpha^{(k)} \mid \beta^{(k)}$ from Gamma $\left(n+a_{0}, b_{0}+\sum_{i=1}^{n} \ln \left(1+\frac{x_{i}}{\beta^{(k)}}\right)\right)$

When the Gibbs sampler achieves approximatively its stationary regime, the sample $\alpha_{N}=$ $\left(\alpha^{(1)}, \ldots, \alpha^{(N)}\right)$ is saved. It is used to compute the Bayesian estimator $\hat{\alpha}$ of $\alpha$. Under the quadratic loss function, we calculate the mean. Recall that our estimator depends on $a_{0}$ and $b_{0}$. So, we note $\hat{\alpha}\left(a_{0}, b_{0}\right)=\operatorname{mean}\left(\alpha_{N}\right)$.

In order to perform the stability Bayesian analysis on $\alpha$, suppose that the prior is not exactly specifed. So, following the methodology of Męczarski and Zieliński (1991), we keep the value $b_{0}$ fixed and consider the parameter $a_{1} \leq a \leq a_{2}$ instead of $a_{0}$ with $a_{1}$ and $a_{2}$ fixed. Then, we obtain the posteriors risk $\operatorname{PR}(a)$ for $\alpha$ when $a$ varies from $a_{1}$ until $a_{2}$,

$$
P R(a)=E\left[\left(\alpha-\hat{\alpha}\left(a_{0}, b_{0}\right)\right)^{2} \mid x\right]=E\left[\alpha^{2} \mid x ; a, b_{0}\right]+\hat{\alpha}^{2}\left(a_{0}, b_{0}\right)-2 \hat{\alpha}\left(a_{0}, b_{0}\right) E\left[\alpha \mid x ; a, b_{0}\right]
$$

Where

$$
\begin{aligned}
E\left[\alpha^{2} \mid x ; a, b_{0}\right] & =\int_{0}^{\infty} \alpha^{2} \pi_{0}\left(\alpha / x ; a, b_{0}\right) d \alpha \\
E\left[\alpha \mid x ; a, b_{0}\right] & =\int_{0}^{\infty} \alpha \pi_{0}\left(\alpha / x ; a, b_{0}\right) d \alpha
\end{aligned}
$$

and the corresponding oscillation of the posterior risk of the Bayesian estimator of $\alpha$ denoted $R 1$

$$
R 1=\left|\max _{a_{1} \leq a \leq a_{2}} P R(a)-\min _{a_{1} \leq a \leq a_{2}} P R(a)\right|
$$

\subsubsection{Bayesian stability for $\beta$}

Now, we use a noninformative prior for $\alpha$ and $\operatorname{Gamma}\left(u_{0}, v_{0}\right)$ prior for $\beta$. The density priors of $\alpha$ and $\beta$ are respectively

$$
\begin{gathered}
\pi_{0}(\alpha)=\frac{1}{\alpha} ; \quad \alpha>0 . \\
\pi_{1}(\beta)=\frac{v_{0}^{u_{0}} \beta^{u_{0}-1} e^{-\beta v_{0}}}{\Gamma\left(u_{0}\right)}
\end{gathered}
$$

Journal home page: www.jafristat.net ; www.projecteuclid.org/as 
The joint posterior distribution of the parameters $\alpha$ and $\beta$ is given by

$$
\pi(\alpha, \beta \mid x) \propto \alpha^{n-1} \beta^{u_{0}-n-1} \exp \left\{-\beta v_{0}-(\alpha+1) \sum_{i=0}^{n} \ln \left(1+\frac{x_{i}}{\beta}\right)\right\}
$$

Here, again, to obtain the marginal distributions of the parameters, we use the Gibbs sampling with Metropolis-Hastings's step and follow the algorithm quoted above but with different parameters of marginal distributions. Then,

$$
\pi(\alpha \mid \beta, x) \propto \alpha^{n-1} \exp \left\{-\alpha \sum_{i=0}^{n} \ln \left(1+\frac{x_{i}}{\beta}\right)\right\} \sim \operatorname{Gamma}\left(n, \sum_{i=0}^{n} \ln \left(1+\frac{x_{i}}{\beta}\right)\right)
$$

and

$$
\pi(\beta \mid \alpha, x) \propto \beta^{u_{0}-n-1} \exp \left\{-\beta v_{0}-(\alpha+1) \sum_{i=0}^{n} \ln \left(1+\frac{x_{i}}{\beta}\right)\right\} \quad \text { is not known }
$$

Following again the methodology of Męczarski and Zieliński (1991) to perform the stability Bayesian analysis on $\beta$, we keep the value $v_{0}$ fixed and consider the parameter $u_{1} \leq u \leq u_{2}$ with $u_{1}$ and $u_{2}$ fixed. Then, we obtain the posteriors risk $P R(u)$ for $\beta$ when $u$ varies from $u_{1}$ until $u_{2}$

$$
P R(u)=E\left[\left(\beta-\hat{\beta}\left(u_{0}, v_{0}\right)\right)^{2} \mid x\right]=E\left[\beta^{2} \mid x ; u, v_{0}\right]+\hat{\beta}^{2}\left(u_{0}, v_{0}\right)-2 \hat{\beta}\left(u_{0}, v_{0}\right) E\left[\beta \mid x ; u, v_{0}\right]
$$

where

$$
\begin{gathered}
E\left[\beta^{2} \mid x ; u, v_{0}\right]=\int_{0}^{\infty} \beta^{2} \pi_{0}\left(\beta / x ; u, v_{0}\right) d \beta \\
E\left[\beta \mid x ; u, v_{0}\right]=\int_{0}^{\infty} \alpha \pi_{0}\left(\alpha / x ; u, v_{0}\right) d \beta
\end{gathered}
$$

and the corresponding oscillation of the posterior risk of the Bayesian estimator of $\beta$ denoted $R 2$

$$
R 2=\left|\max _{u_{1} \leq u \leq u_{2}} P R(u)-\min _{u_{1} \leq u \leq u_{2}} P R(u)\right|
$$

\subsection{Generalized quadratic loss function}

Now, we propose to use the same idea of Larbi and Fellag (2016) and consider generalized quadratic loss function of the form $L(d ; t)=t^{k}(d-t)^{2}$ where $d$ is a Bayesian estimator, $t$ is to be estimated and $k \in Z$ is the parameter of the generalized loss function. Notice that, the basic quadratic loss function is derived for $k=0$.

Now, the oscillation of the PR's depends on $k$ for both $\alpha$ and $\beta$. Our aim is to check if there exist values of $k \neq 0$ such that the oscillation is smaller than we use basic quadratic loss function $(k=0)$.

Journal home page: www.jafristat.net ; www.projecteuclid.org/as 


\subsubsection{Bayesian stability for $\alpha$}

Under the generalized loss function, the Bayesian estimator of $\alpha$ is

$$
\hat{\alpha}\left(a_{0}, b_{0}, k\right)=\frac{E\left[\alpha^{k+1} \mid x\right]}{E\left[\alpha^{k} \mid x\right]}, k \in Z .
$$

The posterior risk for the estimator of $\alpha$ is then

$$
P R(a, k)=E\left[\alpha^{k+2} \mid x ; a, b_{0}\right]-2 \hat{\alpha}\left(a_{0}, b_{0}, k\right) E\left[\alpha^{k+1} \mid x ; a, b_{0}\right]+E\left[\alpha^{k} \mid x ; a, b_{0}\right] \hat{\alpha}\left(a_{0}, b_{0}, k\right)^{2} .
$$

and the oscillation of the posterior risk of $\alpha$ is given by the following formula

$$
R 1(k)=\left|\max _{a_{1} \leq a \leq a_{2}} P R(a, k)-\min _{a_{1} \leq a \leq a_{2}} P R(a, k)\right|
$$

\subsubsection{Bayesian stability for $\beta$}

The Bayesian estimator of $\beta$ is

$$
\hat{\beta}\left(u_{0}, v_{0}, k\right)=\frac{E\left[\beta^{k+1} \mid x\right]}{E\left[\beta^{k} \mid x\right]}, k \in Z .
$$

The posterior risk of the estimator of $\beta$ is given by

$$
P R(a, k)=E\left[\beta^{k+2} \mid x ; u, v_{0}\right]-2 \hat{\beta}\left(u_{0}, v_{0}, k\right) E\left[\beta^{k+1} \mid x ; u, v_{0}\right]+E\left[\beta^{k} \mid x ; u, v_{0}\right] \hat{\beta}\left(u_{0}, v_{0}, k\right)^{2}
$$

and the oscillation of the posterior risk of $\alpha$ is

$$
R 2(k)=\left|\max _{u_{1} \leq u \leq u_{2}} P R(u, k)-\min _{u_{1} \leq u \leq u_{2}} P R(u, k)\right|
$$

The formulas of the estimator, posterior risk and the oscillation are to be approximated numerically in the two cases using MCMC methods.

\section{Monte Carlo study}

\subsection{Bayesian stability for $\alpha$}

First of all, let us propose an example in order to verify if the stationary distribution of the Gibbs algorithm is satisfactory.

\subsubsection{Example}

Consider a sample with size $n=50$ of a $\operatorname{GPD}(0.4,20)$ with $a_{0}=0.8$ and $b_{0}=9$. We perform 1000 iterations with initial value $B[1]=50$. Figure 1 presents the values of $\alpha$ and its mean in each iteration. 

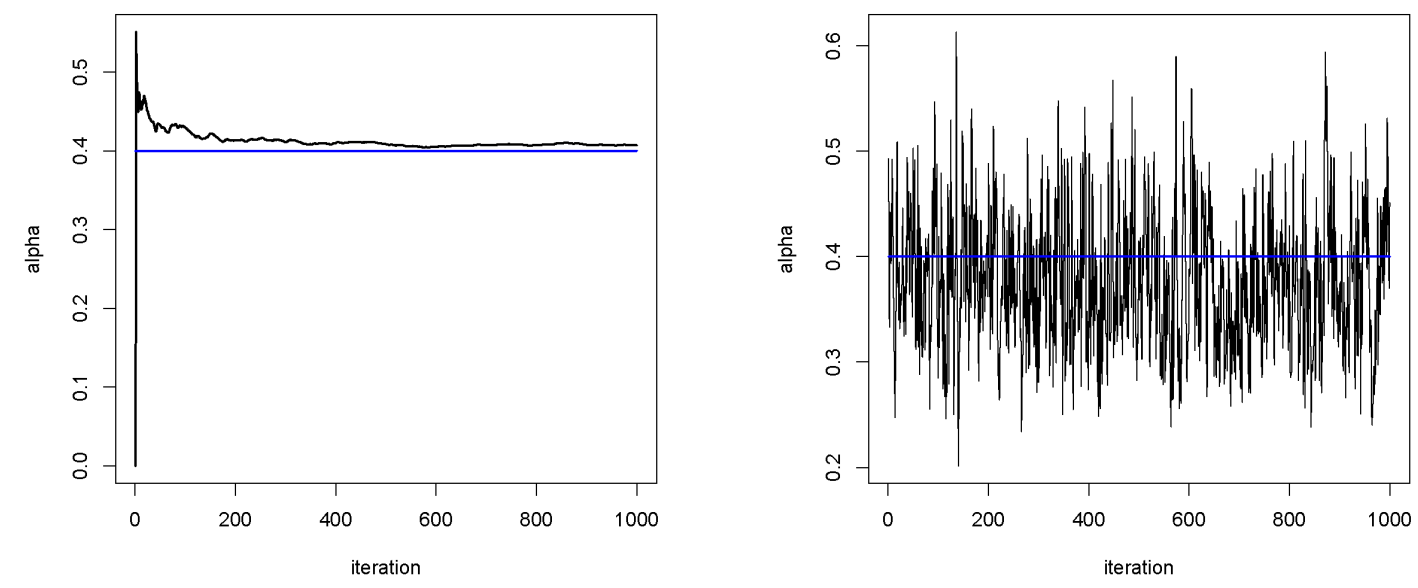

Fig. 1. Variation of the values of $\alpha$. right: using the mean ; left : using Gibbs sampling

Notice that the values of $\alpha$ vary in a reasonable interval around the true value. Also, we remark that the mean stabilizes very quickly at a number close to the true value. So, we can say that the stationary distribution is approximately achieved. Now, consider three samples where $n=10,30,50$ with $a_{0}=0.8$ and $b_{0}=9$ fixed. Table 1 presents the Bayesian estimators of $\alpha$ and their posterior risks for some values of $\alpha$ and $\beta$.

Now, let us study the stability of the Bayesian estimators of $\alpha$. For this, we vary $a$ in the interval $0.5 \leq a \leq 1.5$ and keep $b_{0}=9$ fixed. The Table 2 gives the oscillation of the posterior risk.

Now, let us study the stability of the Bayesian estimators of $\alpha$ under the generalized quadratic loss given above, when $k$ varies. Since the results are invariant with $\beta$, we fix it equal to one, without loss of generality. Then, we restrict the simulation study to $\alpha$ in $\{0.2,0.4,0.6,1\}$. Also, we fix the values $a_{0}=0.8$ and $b_{0}=9$ for the three samples with $n=10,30,50$.

Figure 2, 3 and 4 present the oscillations of the posterior risks for different values of $k$. We notice that the values of the estimator of $\alpha$ are far from the true value for large values of $\alpha$ (see Table 1). Also, one can notice that the oscillation decreases when $n$ increases unless when $\alpha$ is high (see Table 2). We can add that the oscillations are smaller when $\alpha$ is close to zero, and they are invariant with respect to $\beta$. Under the generalized quadratic loss function, we remark that 
F. Mokrani, H. Fellag and A. Necir, Afrika Statistika, Vol. 11(2), 2016, 1061-1074. Robust bayesian inference of generalized Pareto distribution.

\begin{tabular}{|c|c|c|c|c|}
\hline \multirow{2}{*}{$\alpha$} & \multirow{2}{*}{$\beta$} & \multicolumn{3}{|c|}{$\mathrm{n}$} \\
\hline & & 10 & 30 & 50 \\
\hline \multirow{4}{*}{0.2} & 0.2 & $0.1547(0.0025)$ & $0.2410(0.0028)$ & $0.2333(0.0015)$ \\
\hline & 1 & $0.1986(0.0041)$ & $0.2005(0.0017)$ & $0.2008(0.0012)$ \\
\hline & 5 & $0.1376(0.0020)$ & $0.2073(0.0018)$ & $0.2869(0.0025)$ \\
\hline & 20 & $0.3036(0.0113)$ & $0.1853(0.0017)$ & $0.2052(0.0012)$ \\
\hline \multirow{4}{*}{0.4} & 0.2 & $0.4300(0.0265)$ & $0.3032(0.0044)$ & $0.3264(0.0035)$ \\
\hline & 1 & $0.3327(0.0139)$ & $0.5600(0.0190)$ & $0.4596(0.0074)$ \\
\hline & 5 & $0.3505(0.0146)$ & $0.4344(0.0115)$ & $0.4715(0.0089)$ \\
\hline & 20 & $0.3876(0.0189)$ & $0.2611(0.0034)$ & $0.4063(0.0056)$ \\
\hline \multirow{4}{*}{1} & 0.2 & $0.4792(0.0325)$ & $0.5974(0.0303)$ & $0.6318(0.0182)$ \\
\hline & 1 & $0.4855(0.0317)$ & $0.7772(0.0466)$ & $0.7245(0.0264)$ \\
\hline & 5 & $0.4099(0.0249)$ & $0.5528(0.0198)$ & $0.6288(0.0165)$ \\
\hline & 20 & $0.5611(0.0463)$ & $0.5718(0.0217)$ & $0.5940(0.0146)$ \\
\hline \multirow{4}{*}{20} & 0.2 & $1.1850(0.1388)$ & $3.4193(0.3725)$ & $5.6590(0.6387)$ \\
\hline & 1 & $1.2088(0.1401)$ & $1.2099(0.1030)$ & $1.2388(0.1325)$ \\
\hline & 5 & $0.8152(0.0879)$ & $0.9236(0.1358)$ & $1.2553(0.3469)$ \\
\hline & 20 & $0.6521(0.0571)$ & $1.0499(0.0787)$ & $1.7520(0.1229)$ \\
\hline
\end{tabular}

Table 1. Estimators of $\alpha$ with PR's in brackets for $n=10,30,50$ when $a_{0}=0.8 ; b_{0}=9$.

\begin{tabular}{lllll}
\hline \multirow{2}{*}{$\alpha$} & \multirow{2}{*}{$\beta$} & \multicolumn{3}{c}{$\mathrm{n}$} \\
\cline { 2 - 5 } & & 10 & 30 & 50 \\
\hline \multirow{3}{*}{0.2} & 0.2 & 0.0015 & 0.0013 & 0.0006 \\
\cline { 2 - 5 } & 1 & 0.0022 & 0.0013 & 0.0001 \\
\cline { 2 - 5 } & 5 & 0.0013 & 0.0003 & 0.0003 \\
\cline { 2 - 5 } & 20 & 0.0048 & 0.0006 & 0.00008 \\
\hline \hline \multirow{3}{*}{0.4} & 0.2 & 0.0267 & 0.0005 & 0.0004 \\
\cline { 2 - 5 } & 1 & 0.0094 & 0.0088 & 0.0010 \\
\cline { 2 - 5 } & 5 & 0.0242 & 0.0081 & 0.0034 \\
\hline \hline \multirow{3}{*}{1} & 20 & 0.0146 & 0.0003 & 0.0006 \\
\cline { 2 - 5 } & 0.2 & 0.0087 & 0.0058 & 0.0031 \\
\cline { 2 - 5 } & 1 & 0.0150 & 0.0070 & 0.0040 \\
\hline \hline \multirow{3}{*}{20} & 20 & 0.0033 & 0.0026 & 0.0024 \\
\cline { 2 - 5 } & 0.2 & 0.0333 & 0.0864 & 0.1383 \\
\cline { 2 - 5 } & 1 & 0.0355 & 0.3669 & 0.3928 \\
\hline & 20 & 0.1754 & 0.4066 & 0.3588 \\
\hline
\end{tabular}

Table 2. Oscillation of PR's of estimators of of $\alpha$ for $n=10,30,50$ when $a_{0}=0.8 ; b_{0}=9$.

(i). For $\alpha=0.2$, the oscillation decreases when $k$ increases for $n=10,30,50$ (see Figure 2). 

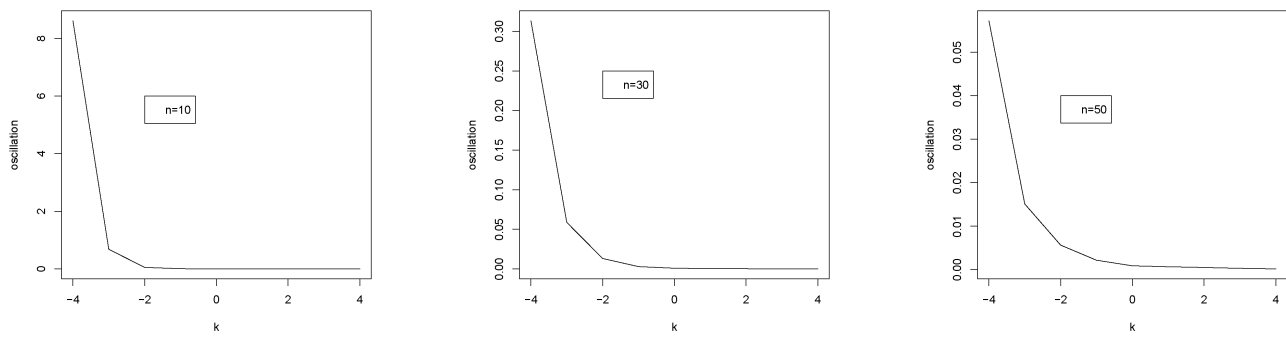

Fig. 2. Variation of the oscillation of the PR's with $k$ for $\alpha$ when $n=10 ; 30 ; 50 ; \alpha=0.2$

(ii). For $\alpha=0.4$, the oscillation values decreases until a particular value $k_{0}$ and then grows for $n=10 ; 30 ; 50$ (see Figure 3).
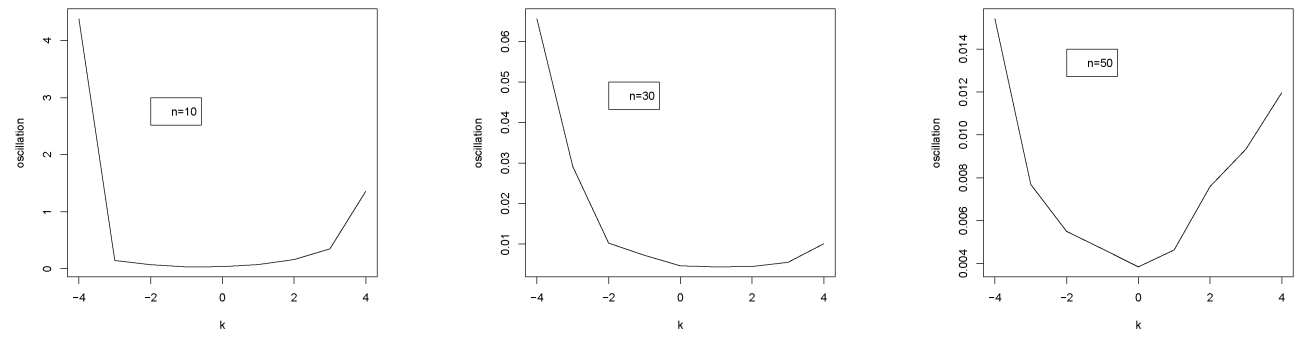

Fig. 3. Variation of the oscillation of the PR's with $k$ for $\alpha$ when $n=10 ; 30 ; 50 ; \alpha=0.4$

(iii). For $\alpha=1$, the oscillation grows when $k$ increases for $n=30,50$, and decreases until some value $k_{1}$ and then grows (see Figure 4).

Then, one can conclude that that for all the studied values of $\alpha$, we can improve the robust Bayesian estimation of $\alpha$. The best situation is obtained when $\alpha$ is small and $k$ enough high.

\subsection{Bayesian stability of $\beta$}

First, as in the previous subsection, let us verify if the stationary distribution of the Gibbs algorithm is approximately achieved in this case. Then, we consider the following example.

Journal home page: www.jafristat.net ; www.projecteuclid.org/as 

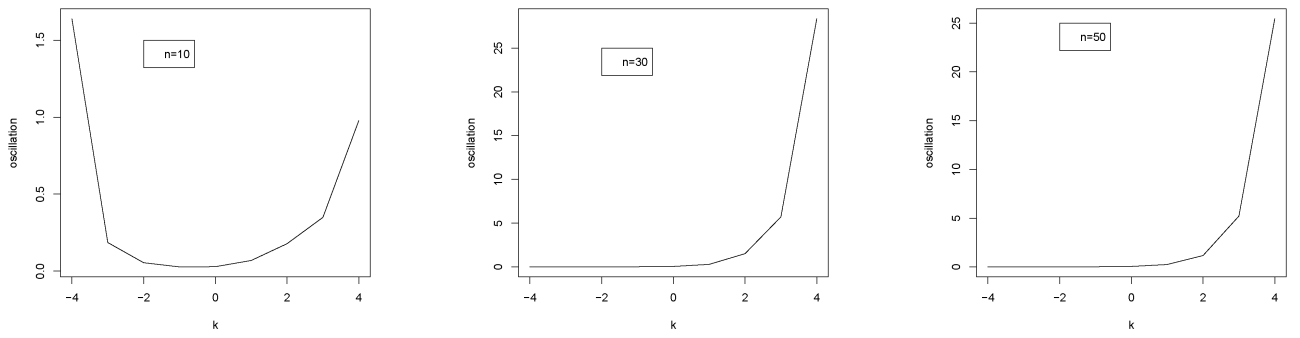

Fig. 4. Variation of the oscillation of the PR's with $k$ for $\alpha$ when $n=10 ; 30 ; 50 ; \alpha=1$

\subsubsection{Example}

Consider a sample with size $n=50$ of a $\operatorname{GPD}(5,0.2)$ with $u_{0}=0.4$ and $v_{0}=5$. Since the conditional distribution $\beta \mid \alpha$ is unknown, we perform 10000 iterations with initial value $B[1]=0.1$. The obtained values of $\beta$ and its mean in each iteration are presented in Figure 5
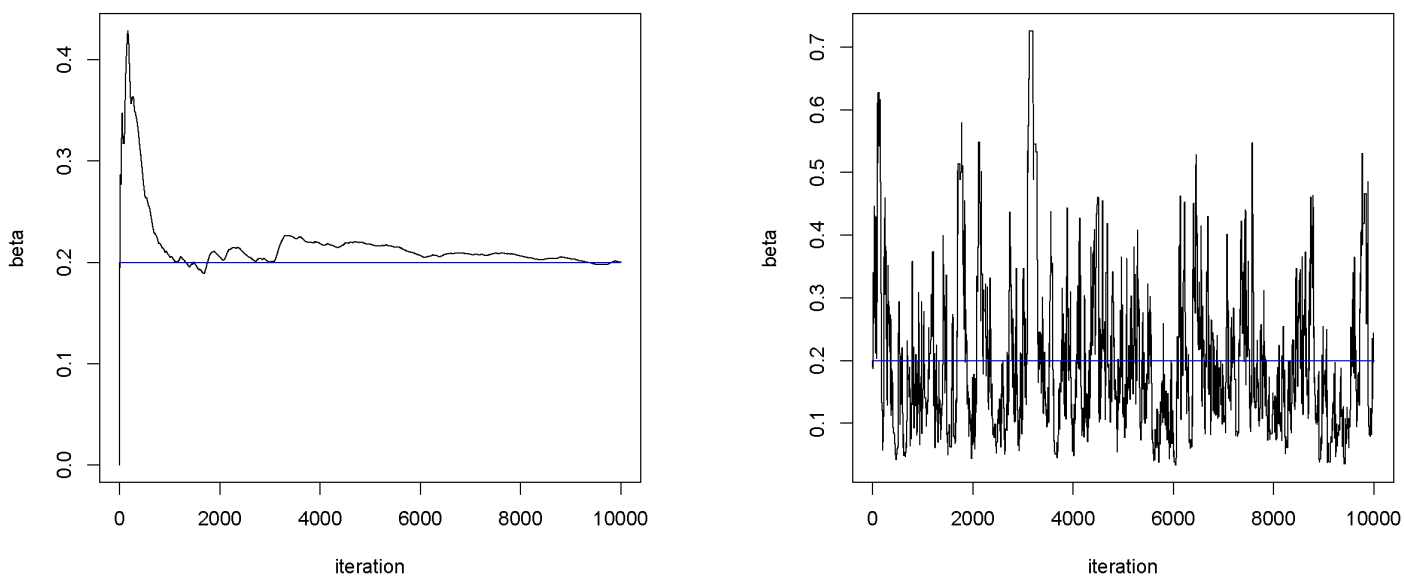

Fig. 5. Variation of values of $\beta$. Right: with his mean; Left: with Gibbs sampling 
F. Mokrani, H. Fellag and A. Necir, Afrika Statistika, Vol. 11(2), 2016, 1061-1074. Robust bayesian inference of generalized Pareto distribution.

\begin{tabular}{lllll}
\hline \multirow{2}{*}{$\beta$} & \multirow{2}{*}{$\alpha$} & \multicolumn{3}{c}{$\mathrm{n}$} \\
\cline { 2 - 5 } & & 10 & 30 & 50 \\
\hline \multirow{3}{*}{0.2} & 0.2 & $0.1595(0.0212)$ & $0.1087(0.0050)$ & $0.2924(0.0251)$ \\
\cline { 2 - 5 } & 1 & $0.0931(0.0089)$ & $0.2009(0.0150)$ & $0.1030(0.0020)$ \\
\cline { 2 - 5 } & 5 & $0.16022(0.0197)$ & $0.1500(0.0273)$ & $0.2220(0.0286)$ \\
\hline \hline \multirow{3}{*}{0.4} & 10 & $0.1805(0.0264)$ & $0.2128(0.0319)$ & $0.3617(0.0965)$ \\
\cline { 2 - 5 } & 0.2 & $0.1431(0.0207)$ & $0.1958(0.0223)$ & $0.2927(0.0207)$ \\
\cline { 2 - 5 } & 5 & $0.2917(0.0382)$ & $0.2730(0.0212)$ & $0.2961(0.0202)$ \\
\hline \hline \multirow{3}{*}{1} & 10 & $0.2220(0.0316)$ & $0.2288(0.0327)$ & $0.2640(0.0230)$ \\
\cline { 2 - 5 } & 0.2 & $0.2879(0.0489)$ & $0.4670(0.0748)$ & $0.6505(0.0671)$ \\
\cline { 2 - 5 } & 1 & $0.2321(0.0334)$ & $0.4716(0.0506)$ & $0.6565(0.0565)$ \\
\hline \hline \multirow{3}{*}{20} & 10 & $0.1967(0.0354)$ & $0.3122(0.0344)$ & $0.5075(0.0589)$ \\
\cline { 2 - 5 } & 0.2 & $0.3439(0.09417)$ & $0.2775(0.0296)$ & $0.4035(0.0471)$ \\
\cline { 2 - 5 } & 1 & $0.5533(0.0745)$ & $1.3863(0.0312)$ & $1.8561(0.4235)$ \\
\cline { 2 - 5 } & 10 & $0.4125(0.0934)$ & $1.0965(0.2054)$ & $1.5682(0.2850)$ \\
\hline
\end{tabular}

Table 3. Estimators of $\beta$ with PR's in brackets for $n=10,30,50$ when $u_{0}=0.4 ; v_{0}=5$.

We notice that the values of $\beta$ vary in a reasonable interval around the true value. Also, we remark that the mean stabilizes very quickly at a number close to the true value. So, we can say that the stationary distribution is approximately achieved. We consider three samples with $n=10,30,50$, for $u_{0}=0.4$ and $v_{0}=5$ fixed. We calculate the Bayesian estimators of $\beta$ and their posterior risks for some values of $\alpha$ and $\beta$. The results obtained are in Table 3 .

Now, in order to study the stability of the Bayesian estimators of $\beta$, we vary $u$ in the interval $0.5 \leq u \leq 1.5$ and keep $v_{0}=5$ fixed. The oscillations of the posterior risk, are given in Table 4 .

Journal home page: www.jafristat.net ; www.projecteuclid.org/as 
F. Mokrani, H. Fellag and A. Necir, Afrika Statistika, Vol. 11(2), 2016, 1061-1074. Robust bayesian inference of generalized Pareto distribution.

\begin{tabular}{lllll}
\hline \multirow{2}{*}{$\beta$} & \multirow{2}{*}{$\alpha$} & \multicolumn{3}{c}{$\mathrm{n}$} \\
\cline { 2 - 5 } & & 10 & 30 & 50 \\
\hline \multirow{3}{*}{0.2} & 0.2 & 0.0693 & 0.0053 & 0.0267 \\
\cline { 2 - 5 } & 1 & 0.0253 & 0.0269 & 0.0027 \\
\cline { 2 - 5 } & 5 & 0.2663 & 0.4172 & 0.3577 \\
\hline \hline \multirow{3}{*}{0.4} & 10 & 0.1669 & 0.2545 & 0.1618 \\
\cline { 2 - 5 } & 0.2 & 0.0556 & 0.0370 & 0.0154 \\
\hline \hline \multirow{3}{*}{1} & 5 & 0.0675 & 0.0201 & 0.0155 \\
\cline { 2 - 5 } & 10 & 0.0752 & 0.1171 & 0.1121 \\
\hline & 0.2 & 0.0725 & 0.0683 & 0.0340 \\
\cline { 2 - 5 } & 1 & 0.0628 & 0.0365 & 0.0306 \\
\hline \hline \multirow{3}{*}{20} & 10 & 0.0854 & 0.0547 & 0.0842 \\
\cline { 2 - 5 } & 0.2 & 0.3024 & 0.4366 & 0.3037 \\
\cline { 2 - 5 } & 1 & 0.2316 & 0.8071 & 0.8361 \\
\hline & 5 & 0.5273 & 0.2700 & 0.2904 \\
\hline
\end{tabular}

Table 4. Oscillation of PR's of estimators of $\beta$ for $n=10,30,50$ when $u_{0}=0.4 ; v_{0}=5$

Now, let us study the stability of the Bayesian estimators of $\beta$ under the generalized quadratic loss given above, when $k$ varies. Since the results are invariant with $\alpha$, we fix $\alpha=1$ without loss of generality. Then, we restrict the simulation study to $\beta \in$ $\{0.08,0.1,0.4,0.6,0.8\}$. Also, we fix the values $u_{0}=0.4$ and $v_{0}=5$ for the three samples with $n=10,30,50$.

Figure 6 and 7 present oscillations of the posterior risks for different values of $k$. We notice that the values of the estimator of $\beta$ are far from the true value for large values of $\beta$ (see Table 3). Also, one can notice that the oscillations are smaller when $\beta$ is close to zero,and they are invariant with respect to $\alpha$ (see Table 4). Under the generalized quadratic loss function, we remark that

(i). For $\beta$ small, the oscillation decreases when $k$ increases for $n=10,30,50$ (see Figure 6 ). 

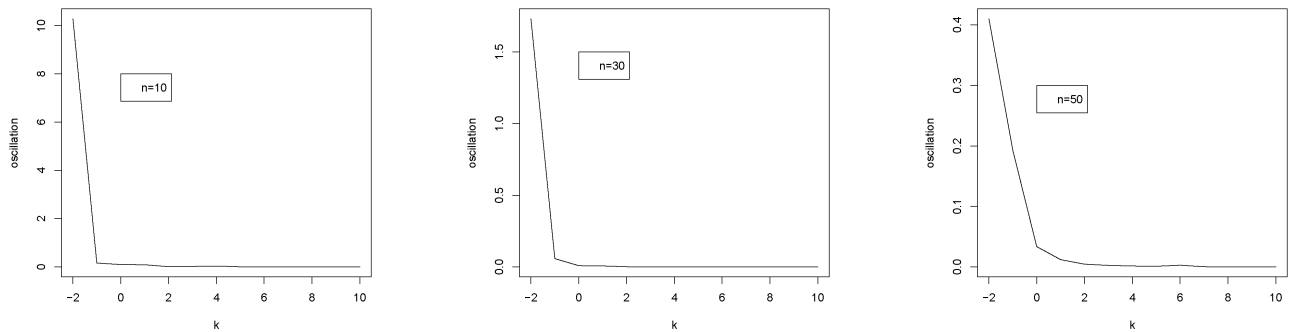

Fig. 6. Variation of the oscillation of the PR's with $k$ for $\beta$ when $n=10 ; 30 ; 50 ; \beta=0.08$

(ii). For $\beta$ higher, the oscillation values grows when $k$ increases for $n=10 ; 30 ; 50$ (see Figure 7).
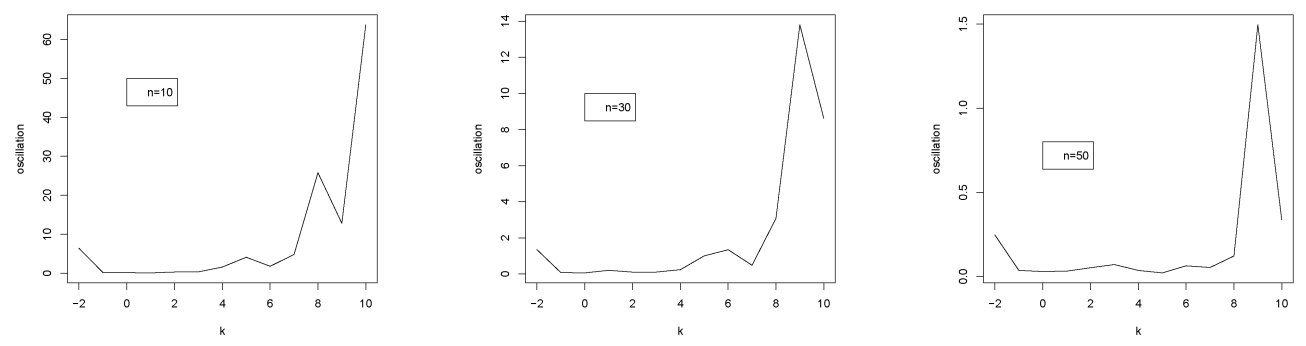

Fig. 7. Variation of the oscillation of the PR's with $k$ for $\beta$ when $n=10 ; 30 ; 50 ; \beta=0.4$

Then, we can say that it is possible to improve the robust Bayesian estimation of $\beta$ when its values are very small.

\section{Conclusion}

In this work, robustness and sensitivity analysis of the Bayesian estimators of a generalized Pareto model is performed according to a specific methodology. Two parameters are considered an the robustness is studied in terms of oscillation of the posterior risk. Then, using an exhaustive Monte Carlo procedure, we proved that, under a suitable generalized loss function, robust estimators can be obtained for the parameters when they are not high.

\section{Acknowledgments}

We thank the referees for their helpful comments.

Journal home page: www.jafristat.net ; www.projecteuclid.org/as 
F. Mokrani, H. Fellag and A. Necir, Afrika Statistika, Vol. 11(2), 2016, 1061-1074. Robust bayesian inference of generalized Pareto distribution.

\section{References}

Arnold, B.C. and Press, S.J.,1989. Bayesian estimation and prediction for Pareto data,J. Amer. Statist. Assoc 84, 1079-1084.

Berger, J.O., Rios Insua, D. and Ruggeri, F., 2000. Bayesian robustness, In Robust Bayesian Analysis (D. Rios Insua and F.Ruggeri, eds.), Springer-Verlag, New York.

De Zea Bermudez, P. and Amaral Turkman, M.A., 2003. Bayesian approach to parameter estimation of the generalized Pareto distribution, TEST 12, 259-277.

De Zea Bermudez, P. and Kotz, S., 2010. Parameter estimation of the generalized Pareto distribution-Part I, J. Statist. Plann. Inference 140, 1353-1373.

Diebolt, J., El-Aroui, M., Garrido, M. and Girard, S., 2005. Quasi-conjugate Bayes estimates for GPD parameters and application to heavy tails modelling, Extremes 8, 57-78.

Gilks, W.R., Richardson, S., Spiegelhalter, D.J., 1986. Markov Chain Monte Carlo Methods in Practice. Chapman and Hall, CRC, London, Boca Raton, FL.

Larbi, L. and Fellag, H., 2016. Robust bayesian analysis of an autoregressive model with exponential innovations. Afrika Statistika, 11(1), 955-964.

Męczarski, M. and Zieliński, R., 1991. Stability of the Bayesian estimator of the Poisson mean under the inexactly specified gamma prior. Statist. Probab. Lett. 12, 329-333.

Micheas A.C., 2006. A Unified Approach to Prior and Loss Robustness, Comm. Statist. Theory Methods, 35, 309-323.

Pickands, J., 1975. Statistical inference using extreme order statistics, Ann. Statist. 3, 119131.

Journal home page: www.jafristat.net; www.projecteuclid.org/as 